\title{
Cuidados paliativos em terapia intensiva: uma revisão integrativa de literatura
}

\section{Palliative measures in intensive care: an integrative literature review}

\author{
Tiago Lima Barreto ${ }^{1}$, Lidiele Roque Bueno ${ }^{2}$, Jéssica Delamuta Vitti ${ }^{3}$, Nelson Francisco Serrão \\ Junior $^{2}$ \\ 1 - Faculdade Inspirar, Bauru, SP, Brasil. \\ 2 - Universidade Federal do Pampa - UNIPAMPA, Uruguaiana, RS, Brasil. \\ 3 - Instituto Educacional Campos, Campinas, SP, Brasil.
}

\begin{abstract}
RESUMO
Introdução: a terapêutica em conjunto com o avanço tecnológico dentro da unidade de terapia intensiva (UTI) fez com que doenças fatais se tornassem crônicas, aumentando o uso de procedimentos invasivos na tentativa de manutenção da vida, propiciando a distanásia. Os cuidados

tlbarreto.fisio@yahoo.com. paliativos (CP) são uma alternativa para impedir a distanásia, com princípios pautados no trabalho multidisciplinar e abordagem do cuidado frente à dignidade da finitude humana. Objetivo: analisar por meio de uma revisão integrativa da literatura, o que vem sendo discutido sobre os benefícios da abordagem dos CP dentro da UTI adulto, quais as suas limitações atuais e quais os protocolos utilizados nos últimos cinco anos. Método: por meio de revisão integrativa de literatura, foi realizado estratégia de busca com descritores nos portais The National Library of Medicine e Biblioteca Virtual em Saúde, analisados artigos científicos no período de 2014 a 2018, nos idiomas português, inglês e espanhol. Resultados: Foram identificados 26 trabalhos, e após o processo de triagem e seleção, foram elegíveis para o presente estudo apenas 12 artigos. Os benefícios dos CP em UTI estão atrelados à diminuição significativa nos procedimentos

Palavras-chave: invasivos, eficácia no planejamento de cuidados, aumento na qualidade do processo de morrer, Cuidados Paliativos; Uni- além de redução de gastos hospitalares para o sistema de saúde. Conclusão: a abordagem dos CP dade de Terapia Intensi- tem impacto positivo na UTI, mas ainda enfrenta limitações inerentes à falta de estudos de maior va; Respiração Artificial. impacto, assim como protocolos assistenciais pautados em evidências.
\end{abstract}

\section{ABSTRACT}

Introduction: therapies, along with technological advances within the intensive care units (ICU), have caused fatal diseases to become chronic, increasing the use of invasive procedures in an attempt to maintain life, providing dysthanasia. Palliative care (PC) is an alternative to prevent dysthanasia, with principles based on multidisciplinary work and approach to care in face of the dignity of human finitude. Objective: to analyze, through an integrative literature review, what has been discussed about the benefits of the PC approach within the adult ICU, its current limitations and the protocols used in the last five years. Method: through an integrative literature review, a search strategy was performed with descriptors on the databases of The National Library of Medicine and Virtual Health Library portals, analyzing scientific articles from 2014 to 2018, in Portuguese, English and Spanish. Results: 26 papers were identified, of which only 12 were eligible for the present study, after the screening and selection process. The benefits of $\mathrm{PC}$ in the ICU are linked to a significant decrease in invasive procedures, effectiveness in care

Keywords: planning, increase in the quality of the dying process, in addition to reducing hospital expenses Palliative Care; Intensi- for the health system. Conclusion: the PC approach has a positive impact on the ICU, but still ve Care Unit; Artificial faces limitations inherent to the lack of studies with greater impact, as well as evidence-based Respiration. care protocols. 


\section{INTRODUÇÃO}

A Unidade de Terapia Intensiva (UTI) é descrita como unidade de internação de pacientes graves, que necessitam de atenção integral e contínua de profissionais especializados, materiais e tecnologias relacionadas ao diagnóstico, monitorização e tratamento ao paciente. ${ }^{1}$

$\mathrm{O}$ avanço das tecnologias utilizadas em UTI aumenta a perspectiva terapêutica em diversas situações clínicas, porém, tal evolução não é capaz de garantir o alívio do sofrimento humano, e com isso, promove o prolongamento da vida por meio de tratamentos não curativos. $\mathrm{O}$ avanço tecnológico na área da saúde é amplamente valorizado, mas pouco se fala sobre a arte da humanização em saúde. ${ }^{2}$

A terapêutica em conjunto com o avanço tecnológico, fez com que doenças consideradas fatais se transformassem em doenças crônicas, propiciando a longevidade da população, além do aumento da expectativa de vida e a crescente longevidade da população idosa brasileira. Com isso, estes pacientes sem prognóstico de cura concentramse nos hospitais, recebendo assistência inadequada, quase sempre pautada na tentativa de cura por meio de métodos invasivos e de alta tecnologia, sem que haja um planejamento e desenvolvimento de políticas públicas voltadas para a assistência em saúde desta população. ${ }^{3,4}$

O prolongamento desnecessário do processo de morte por meio de tratamentos artificiais e pouco efetivos, que por algum fator acarreta no sofrimento e altera o processo natural de morrer, sem qualidade e sem dignidade, é compreendido como distanásia. A distanásia é o excesso de procedimentos que promovem o prolongamento da vida biológica, é uma prática contrária à eutanásia, que é entendida como processo de abreviação do tempo de vida de uma pessoa. Vale ressaltar que no Brasil, a prática de eutanásia é considerada crime pela legislação penal. ${ }^{4}$

Em contrapartida, a ortotanásia não dispõe de ações e práticas de abreviação ou de prolongamento do processo de morte, atua na assistência integral ao paciente com abordagens terapêuticas direcionadas ao alívio do sofrimento, proporcionando uma morte natural e respeitando a dignidade humana na finitude da vida. ${ }^{4} \mathrm{O}$ conceito de $\mathrm{CP}$ teve origem na Inglaterra por meio do movimento hospice, idealizado por Dame Cecily Saunders, que descreve a filosofia do cuidado da pessoa em processo de finitude, com foco em aliviar o sofrimento físico, psíquico, espiritual e social, pautado na dignidade humana. ${ }^{5}$

A Organização Mundial de Saúde $^{5}$ define cuidados paliativos $(\mathrm{CP})$ como cuidado ativo e total para pacientes cuja doença não responde a tratamentos que promovem cura, por meio de uma abordagem que melhora a qualidade de vida dos pacientes e familiares que estão enfrentando problemas associados à doenças potencialmente fatais, atuando com prevenção e alívio do sofrimento. ${ }^{6}$

No Brasil, os CP tiveram início em 1980, com crescimento importante a partir do ano 2000 , em detrimento da consolidação de serviços pioneiros já existentes. ${ }^{3}$ A revista Britânica The Economist Intelligence Unit (2015) publicou o ranking dos países com qualidade durante o processo de morte, em que o Brasil ocupa a $42^{a}$ colocação, ficando atrás de países como Hungria, Equador e Uruguai. Quanto a capacidade de promover abordagens em CP, o Brasil ocupa a $64^{a}$ posição, ficando evidente que o Brasil tem muito que avançar quando se fala sobre $\mathrm{CP}$ e humanização no processo de morte. ${ }^{7}$

Os CP atualmente são discutidos como uma alternativa para impedir a distanásia e a eutanásia, seu princípio é embasado em trabalho multidisciplinar, com abordagem do cuidado frente à realidade do fim da vida humana. ${ }^{2}$ Contudo, os CP não estão relacionado à eutanásia, e, por ainda serem confundidos com tal prática, causa equívocos nas tomadas de decisões quanto à realização de intervenções em pacientes que poderiam se beneficiar desta terapêutica, somado a dificuldade em definir a linha tênue do "fazer" e do "não fazer". 3

$\mathrm{Na}$ UTI ainda se confunde a visão e entendimento de CP com o cuidado de fim de vida (CFV), essa concepção errônea de que CPé sinônimo de paciente terminal pode ser atribuída por uma carência de informação e entendimento dos profissionais, que também apresentam dificuldades em definir quais pacientes são elegíveis para $\mathrm{CP}$ e quais terapêuticas devem ser adotadas nesse contexto. ${ }^{8}$

Os CP podem parecer contraditórios frente a uma unidade que possui amplo perfil tecnológico como a UTI, mas, os cuidados intensivos atualmente devem estar voltados tanto para prevenção, diagnóstico, monitoramento e tratamento, quanto para a paliação. ${ }^{9}$

É necessária abordagem interdisciplinar no campo dos $\mathrm{CP}$, gerenciada por uma equipe multiprofissional junto à família. O fisioterapeuta, por meio de avaliação específica e tratamento adequado, 
com objetivo voltado ao alívio do sofrimento, oferece suporte aos pacientes com repercussão no conforto, qualidade de vida, dignidade, além de auxiliar os familiares na assistência ao paciente. ${ }^{3}$

O presente estudo tem como objetivo analisar, por meio de uma revisão integrativa da literatura, o que vem sendo discutido sobre os benefícios da abordagem dos CP nas UTI adulto, quais as suas limitações atuais e quais os protocolos utilizados nos últimos cinco anos.

\section{MÉTODO}

Trata-se de um estudo de natureza descritiva, com metodologia de revisão integrativa da literatura. Foram analisados artigos científicos nos idiomas português, inglês e espanhol, que contemplavam assuntos de interesse voltados para abordagem terapêutica de CP em UTI adulto.

Foram utilizados os portais The National Library of Medicine (NCBI) e Biblioteca Virtual em Saúde (BVS), nos quais foram acessadas as seguintes fontes de pesquisa: Scientific Electronic Library Online (SciELO), PubMed, IBCS e Medline.

A estratégia de busca foi embasada nos descritores DeCS (Descritores em Ciências da Saúde), em português e suas variações em inglês e espanhol, descritos a seguir: "cuidados paliativos", "unidade de terapia intensiva", "cuidados paliativos na terminalidade da vida" e " respiração artificial"; em inglês: "palliative care", "intensive care unit", "hospice care" e, "artificial respiration"; e, em espanhol: "cuidados paliativos", "unidades de cuidados intensivos", "cuidados paliativos al final de la vida", "respiración artificial".

Os descritores MeSh empregados e suas respectivas variações foram: "Palliative Care", "Palliative Therapy", "Palliative Treatment", "Palliative Treatments", "Palliative Surgery", "Intensive Care Unit", "Hospice Care”, "Hospice Programs", "Hospice Program”, "Bereavement Care", "Artificial Respiration", "Artificial Respirations", "Mechanical Ventilation" e "Mechanical Ventilations".

A revisão integrativa da literatura foi realizada no ano de 2019 e foram selecionados artigos publicados nos últimos cinco anos, de 2014 a 2018, utilizando os seguintes critérios de inclusão: Trabalhos nos idiomas português, inglês e espanhol, estudos realizados em pacientes adultos (maiores de 18 anos) com tema envolvendo CP em UTI e estudos com abordagens multidisciplinares dos CP.

Foram excluídos os trabalhos duplicados encontrados em diferentes bases de dados, trabalhos na área de pediatria e neonatologia (pacientes menores de 18 anos), estudos envolvendo uma única área profissional dos $\mathrm{CP}$ e estudos que não possuíam como tema os CP e/ou UTI.

$\mathrm{O}$ processo de elegibilidade dos artigos compreendeu as seguintes etapas: 1) identificação, 2) triagem, 3) seleção e 4) elegibilidade. Na etapa de identificação, foi realizada a estratificação dos artigos nas bases de dados após busca com os descritores nos períodos de 2014 a 2018. A triagem foi executada a partir da leitura e análise dos títulos e resumos dos artigos identificados, aplicando os critérios de exclusão. A etapa de seleção esteve relacionada à leitura integral dos artigos estratificados após a triagem e identificação dos artigos elegíveis.

\section{RESULTADOS}

Foram identificados 26 artigos a partir da estratégia de busca nos portais citados. Após a triagem dos artigos por meio de análise de títulos e resumos, permaneceram 16 artigos. Destes, 4 foram excluídos no processo de seleção, por meio de leitura integral dos trabalhos, que não contemplavam abordagem de CP e UTI como tema central. Foram designados 12 artigos elegíveis após processo de seleção e analise dos critérios de exclusão, como demonstrado na Figura 1.

Figura 1- Fluxograma de triagem do estudo e seleção dos artigos.

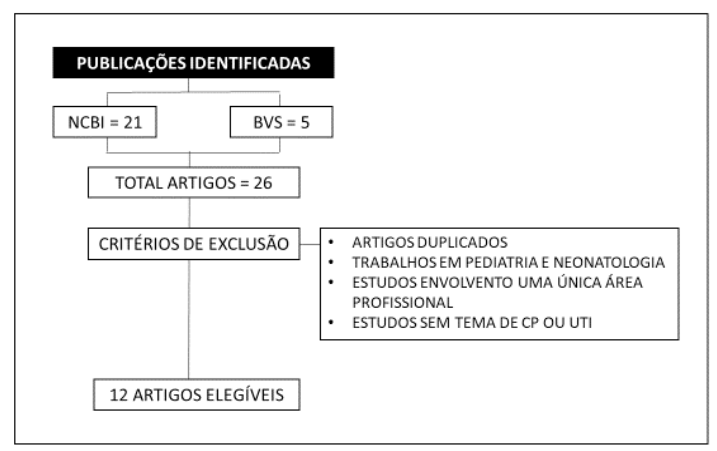

$\mathrm{CP}=$ Cuidados paliativos; $\mathrm{UTI}=$ Unidade de terapia intensiva; $\mathrm{NCBI}=$ The national library of medicine; $\mathrm{BVS}=$ Biblioteca virtual em saúde. 
Dos 12 artigos elegíveis, são 4 estudos observacionais retrospectivos, 3 estudos coorte retrospectivos, 3 revisões narrativas, 1 revisão sistemática e 1 estudo coorte prospectivo. A maior parte dos estudos são relacionados à abordagem de pacientes com diagnóstico de câncer em fase terminal.

Os estudos observacionais e tipo coorte foram agrupados e estão alocados na tabela 1 . Os estudos de revisão narrativa e revisão sistemática estão alocados na tabela 2 .

\section{DISCUSSÃO}

$\mathrm{O}$ presente estudo utilizou 12 artigos que abordaram assuntos relacionados a $\mathrm{CP}$ e terminalidade da vida em UTI adulto, destes foram identificados 4 estudos observacionais retrospectivos, 3 estudos coorte retrospectivos, 3 revisões narrativas, 1 revisão sistemática e 1 estudo coorte prospectivo. Observouse que 5 estudos ( 2 estudos coorte e 3 estudos observacionais retrospectivo) estiveram relacionados à abordagem de pacientes com diagnóstico de câncer em fase terminal. Os estudos com objetivo principal de orientar o processo de retirada de suporte artificial de vida (SAV) foram exclusivamente revisões narrativas.

A comunicação entre a equipe interdisciplinar nos cuidados prestados ao paciente demonstrou ser um dos mecanismos fundamentais para obter sucesso nas tomadas de decisões, mas atualmente apresenta grandes falhas por parte dos profissionais. ${ }^{10,11}$ Esses achados sobre comunicação corroboram com o estudo de Santos et al., ${ }^{12}$ que apesar do reconhecimento da importância dos CP pelos profissionais, os mesmos apresentam carências nas comunicações entre equipe.

A equipe interdisciplinar na assistência de CP na UTI composta por médico enfermeiro e fisioterapeuta, deve orientar nas tomadas de decisões, bem como na retirada da ventilação mecânica invasiva (VMI), sendo esta que traduz grande dificuldade física e emocional por parte dos profissionais. ${ }^{13,14}$ Corroborando aos achados do presente estudo, para alguns autores, ${ }^{12,15,16}$ o motivo pelo qual estes profissionais apresentam esta dificuldade está relacionado a carência na formação a respeito dos CP e a supervalorização dada aos cuidados técnicos, em detrimento de um cuidado envolvendo as dimensões psicossocioespirituais, interferindo significativamente no planejamento e direcionamento de cuidados dos pacientes fora de possibilidade de cura.
Observa-se que pacientes mais jovens são mais suscetíveis a receber VMI e permanecer na UTI, e menos propensos a receber consulta da especialidade de CP. A não adoção de SAV está relacionada pacientes com idade avançada, declínio funcional e pior prognóstico. ${ }^{17-20}$ Estes que corroboram com estudos encontrados na literatura. ${ }^{16,22}$

Constatou-se no presente estudo que pacientes com diagnóstico de câncer são mais elegíveis a receber consulta dos $\mathrm{CP}$ precocemente e menos propensos a receber tratamento invasivo quando comparado a outras patologias. ${ }^{21}$ Corroborando com estudos que evidenciaram que diagnóstico de câncer atualmente vem sendo abordado como a principal indicação de CP em UTI. ${ }^{22}$

A presença de demência demonstrou ser um fator que influencia na tomada de decisões dos profissionais de saúde, tornando os pacientes com demência mais suscetíveis a receber tratamentos invasivos. ${ }^{23}$ Esses achados corroboram com o estudo de Santos et al..$^{24}$ que explanam a heterogeneidade e imprevisibilidade de pacientes portadores de demência como uma barreira que dificulta a avaliação dos critérios para o acesso aos CP.

Os benefícios da abordagem de CP em UTI contribuem com redução de gastos hospitalares ao Sistema de Saúde, além de ser um recurso factível de fornecer cuidados de fim de vida (CFV) de qualidade para pacientes internados em UTI, decorrente de diminuição no uso de VMI e outros procedimentos invasivos como traqueostomia e gastrostomia. ${ }^{17,25}$ Esses dados vêm ao encontro com a literatura que aponta benefícios da abordagem de CP em UTI, proporcionando diminuição do uso de $\mathrm{SAV}$, aumento na qualidade dos CFV e preservação da dignidade humana. ${ }^{22}$

Embora não haja evidências se o SAV deve ser retirado simultaneamente ou seguir uma sequência específica, no estudo de Delaney e Downar ${ }^{10}$ descrevem que a extubação da VMI deve ser o último suporte a ser retirado e seu processo de desmame consiste na redução gradual da oferta de oxigênio. ${ }^{14}$ Refutando tais achados, a Academia Nacional de Cuidados Paliativos (ANCP) descreve que CP não devem ser baseados em protocolos e que o manejo do SAV, no fim de vida, deve visar o conforto, evitando a obstinação terapêutica, e que por sua vez, não há uma fórmula específica de manejo no SAV. ${ }^{3}$ Autores reiteram que não há uma abordagem técnica única, e que a descontinuação do SAV, seja imediata ou 
Tabela 1- Apresentação dos estudos observacionais e estudos de coorte.

\begin{tabular}{|c|c|c|c|}
\hline Autor, ano & Objetivos & Tipo de estudo/Método & Resultados e conclusão \\
\hline $\begin{array}{l}\text { Nakagawa et al., } \\
2018\end{array}$ & $\begin{array}{l}\text { Analisar as diferenças dos CFV e efeito dos CP } \\
\text { entre pacientes com dispositivos de assistência } \\
\text { ventricular e pacientes transplantados }\end{array}$ & $\begin{array}{l}\text { Coorte retrospectivo/ Análise de prontuários no período de } \\
2010 \text { a 2016, avaliado marcadores de CFV como: local da } \\
\text { morte, ordem de reanimação, uso de SAV (VMI, diálise e } \\
\text { nutrição artificial). }\end{array}$ & $\begin{array}{l}\text { As consultas com CP foram menos frequentes no grupo transplante. Já o óbito em UTI foi mais frequente nos } \\
\text { pacientes transplantados. Os dados demonstram que a maioria dos pacientes morrem na UTI em uso de SAV. } \\
\text { A preferência de não ser reanimado esteve relacionada à melhor consciência do prognóstico, idade avançada, e } \\
\text { declínio funcional. Com a abordagem de CP em UTI foram menos frequentes o uso de VMI bem como outros SAV. } \\
\text { Conclusão: É necessário uma equipe multidisciplinar em CP orientando nas tomadas de decisões }\end{array}$ \\
\hline Hua et al., 2018 & $\begin{array}{l}\text { Avaliar a disponibilidade de CP, a eficácia } \\
\text { dos CP em pacientes críticos e a diminuição } \\
\text { de marcadores de tratamento intensivo em } \\
\text { pacientes internados na UTI }\end{array}$ & $\begin{array}{l}\text { Coorte retrospectivo/ Análise de dados do sistema } \\
\text { cooperativo de planejamento e pesquisa do estado de New } \\
\text { York à nível hospitalar entre 2008-2014. Analisado tempo } \\
\text { de internação, disponibilidade de CP, uso de SAV incluindo } \\
\text { traqueostomia e RCP, alta hospitalar e mortalidade. }\end{array}$ & $\begin{array}{l}\text { Identificados } 151 \text { hospitais em New York, destes, somente } 86 \text { com programa de CP, e eram mais propensos a } \\
\text { serem hospitais universitários. A realização de traqueostomia e gastrostomia foi mais frequente em hospitais sem } \\
\text { programa de CP. Houve associação significativa entre alta hospitalar nos hospitais com programa de CP quando } \\
\text { comparados aos hospitais sem CP. O uso de CP hospitalar não foi associado à diferença significativa na utilização de } \\
\text { tratamentos intensivos } \\
\text { Conclusão: A disponibilização de CP hospitalar esteve associado à aumento significativo na utilização do } \\
\text { programa para pacientes em UTI. }\end{array}$ \\
\hline Huang et al., 2017 & $\begin{array}{l}\text { Comparar os cuidados prestados à pacientes } \\
\text { com câncer em fase terminal com demência e } \\
\text { sem demência, durante os últimos meses antes } \\
\text { da morte. }\end{array}$ & $\begin{array}{l}\text { Coorte retrospectivo/ Análise de banco de dados } \\
\text { governamental no período de } 2000 \text { a } 2012 \text { com pacientes } \\
\text { com diagnóstico de câncer. Foram comparados os cuidados } \\
\text { entre } 1^{\circ} \text { e } 3^{\circ} \text { mês antes da morte entre os dois grupos, bem } \\
\text { como tipo de internação, uso de quimioterapia, RCP, IOT, } \\
\text { VMI e exames diagnóstico avançados. }\end{array}$ & $\begin{array}{l}\text { Pacientes com câncer e demência receberam mais procedimentos invasivos como RCP, IOT, VMI e sonda de } \\
\text { alimentação e mais propensos a internações em UTI, porém menos quimioterapia e exames diagnóstico avançado } \\
\text { quando comparados aos pacientes com câncer e sem demência. } \\
\text { Para os médicos não há benefícios para busca de exames avançados em pacientes com demência em fase } \\
\text { terminal, em contrapartida o uso de SAV foi frequente nesse grupo. }\end{array}$ \\
\hline Wright et al., 2014 & $\begin{array}{l}\text { Determinar se o recebimento de quimioterapia } \\
\text { em pacientes com câncer em estado terminal } \\
\text { está associado à cuidados invasivos e UTI } \\
\text { como local de óbito desses pacientes. }\end{array}$ & $\begin{array}{l}\text { Coorte Prospectivo/ Estudo multicêntrico, de pacientes } \\
\text { recrutados com câncer metastático e prognóstico de } 6 \\
\text { meses de vida, de } 10 / 2002 \text { a } 02 / 2008 \text { e que foram à óbito } \\
\text { durante o período do estudo. Avaliado localização da morte, } \\
\text { tempo de tratamento nos CP. }\end{array}$ & $\begin{array}{l}\text { A coorte foi de } 386 \text { pacientes, os pacientes mais jovens eram mais propensos a receber quimioterapia paliativa e } \\
\text { menos propensos a reconhecer seu estado terminal. Os que receberam quimioterapia paliativa foram mais propensos a } \\
\text { RCP e VMI na última semana de vida, e de encaminhamentos tardios aos CP, maior probabilidade de morrer em UTI. } \\
\text { Conclusão: Sugere menor uso de quimioterapia em pacientes com câncer com expectativa menor que } 6 \text { meses, } \\
\text { para reduzir os riscos com cuidados invasivos e melhorar a qualidade dos CFV. }\end{array}$ \\
\hline Binney et al., 2014 & $\begin{array}{l}\text { Analisar a viabilidade da transferência da UTI } \\
\text { para CP e seu impacto econômico; descrever } \\
\text { as características clínicas dos pacientes } \\
\text { transferidos aos CP comparando com os que } \\
\text { permanecem na UTI. }\end{array}$ & $\begin{array}{l}\text { Estudo observacional retrospectivo/ Analise de prontuário } \\
\text { de pacientes internados em UTI de pacientes adultos, que } \\
\text { morreram em UTI e CP de } 2 \text { centros médicos americanos } \\
\text { em um período de } 6 \text { meses. }\end{array}$ & $\begin{array}{l}\text { Os pacientes transferidos da UTI para CP eram significativamente mais velhos, pacientes jovens permaneceram } \\
\text { na UTI e eram mais propensos ao uso de VMI, vasopressor, e menos propensos a receber consulta com CP. O impacto } \\
\text { econômico com CP em UTI foi de US\$1.384.110 evitados com terapias invasivas em } 6 \text { meses. } \\
\text { Conclusão: CP são uma forma viável de fornecer CFV de qualidade para pacientes em UTI, além de redução de } \\
\text { custos hospitalares e Sistema de Saúde em geral. }\end{array}$ \\
\hline Gu et al., 2016 & $\begin{array}{l}\text { Investigar os fatores e as características } \\
\text { associadas ao processo decisório no tratamento } \\
\text { de sustentação de vida em pacientes com câncer } \\
\text { em fase terminal. }\end{array}$ & $\begin{array}{l}\text { Estudo observacional retrospectivo/ Análise de sistema } \\
\text { de registro médico da Universidade de Fudan Shanghai } \\
\text { Cancer Center, de pacientes com câncer terminal que } \\
\text { morreram entre } 2007 \text { a } 2013 \mathrm{em} \mathrm{CP.}\end{array}$ & $\begin{array}{l}\text { Os } 436 \text { pacientes com câncer avançado foram a óbito no período do estudo, } 40 \% \text { dos pacientes permaneceram } \\
\text { com nutrição artificial até a morte; } 6 \% \text { receberam RCP sem sucesso; } 49 \% \text { receberam vasopressores; apenas } 2 \text { pacientes } \\
\text { receberam VMI e } 1 \text { traqueostomia, os pacientes com idade avançada tinham menor probabilidade de receber SAV. O } \\
\text { intervalo de tempo entre a decisão de CP até o óbito foi de } 20 \text { horas. }\end{array}$ \\
\hline Koff et al., 2017 & $\begin{array}{l}\text { Explorar as diferenças na utilização de SAV e } \\
\text { dos CFV de pacientes que morreram na UTI } \\
\text { com câncer em comparação com aqueles sem } \\
\text { câncer. }\end{array}$ & $\begin{array}{l}\text { Estudo observacional retrospectivo/ Análise de banco } \\
\text { de dados do Hospital universitário Thomas Jefferson da } \\
\text { Filadélfia. Examinado SAV, comunicação no fim de vida, } \\
\text { frequência de uso de intervenções invasivas, duração de } \\
\text { VMI e tempo de permanência. }\end{array}$ & $\begin{array}{l}\text { A VMI foi mais frequente entre pacientes sem câncer e foi usado por um período maior quando comparado aos } \\
\text { pacientes com câncer. As consultas com CP foram menos frequentes em pacientes sem câncer. } \\
\text { Pacientes com câncer recebem menos tratamentos invasivos na UTI e fizeram transição precoce aos CP com } \\
\text { melhor qualidade no processo de fim de vida quando comparados aos pacientes sem câncer. } \\
\text { Conclusão: A maior compreensão da terminalidade do câncer, provoca planejamento antecipado para os CFV, } \\
\text { levando a um tendenciamento na abordagem dos cuidados. }\end{array}$ \\
\hline Ersek et al., 2017 & $\begin{array}{l}\text { Examinar as relações entre o tratamento } \\
\text { agressivo nos últimos } 30 \text { dias de vida e as } \\
\text { avaliações das famílias enlutadas de pacientes } \\
\text { com câncer em CFV }\end{array}$ & $\begin{array}{l}\text { Estudo observacional retrospectivo/ Selecionado pacientes } \\
\text { que foram a óbito em UTI, hóspice, e Unidade de CP entre } \\
2010 \text { e } 2012 \text {, avaliado o uso de VMI e quimioterapia nos } \\
\text { últimos } 30 \text { dias, bem como o questionário BFS (Bereaved } \\
\text { Family Survey) }\end{array}$ & $\begin{array}{l}\text { Os pacientes com idade menor que } 65 \text { anos eram mais propensos a receber medidas invasivas como quimioterapia } \\
\text { e VMI e morrer em UTI. Os } 70 \% \text { dos pacientes receberam pelo menos } 1 \text { tratamento invasivo. Os escore do questionário } \\
\text { BFS foram mais baixos para aqueles pacientes que receberam medidas invasivas. } \\
\text { Conclusão: As preferências para tratamentos agressivos estão associados à pacientes mais jovens. Os cuidados } \\
\text { agressivos não contribui positivamente no desfecho de fim de vida e são indicadores de CFV de baixa qualidade. }\end{array}$ \\
\hline
\end{tabular}

$\mathrm{CFV}=$ Cuidados de fim de vida; $\mathrm{CP}=$ Cuidados paliativos; $\mathrm{SAV}=$ Suporte artificial de vida; VMI= Ventilação mecânica invasiva; UTI= Unidade de terapia intensiva; $\mathrm{RCP}=$ Reanimação cardiopulmonar; IOT= Intubação orotraqueal; BFS= Bereaved Family survey.

Rev. Interdisciplin. Promoç. Saúde - RIPS, Santa Cruz do Sul, 3(2):75-82, abr/jun. 2020 ISSN: 2595-3664 
Tabela 2 - Apresentação dos estudos de Revisão Narrativa e Sistemática.

\begin{tabular}{|c|c|c|c|}
\hline Autor, ano & Objetivos & $\begin{array}{l}\text { Tipo de estudo/ } \\
\text { Método }\end{array}$ & Resultados e conclusão \\
\hline Bodnar, 2018 & $\begin{array}{l}\text { Analisar as diferenças } \\
\text { na realização do } \\
\text { procedimento de retirada } \\
\text { da VMI entre unidades de } \\
\text { CP e UTI }\end{array}$ & Revisão Narrativa & $\begin{array}{l}\text { A retirada da VMI deve ser preferencialmente realizada pela equipe de CP, com gerenciamento no alívio dos sintomas, de } \\
\text { preferência em um ambiente restrito e sereno, tal equipe deve ser interdisciplinar, e se faz necessário a presença da equipe da UTI } \\
\text { para fornecer feedback de apoio ao paciente e familiar. } \\
\text { Deve-se usar opióides como midazolam e propofol para alívio de sintomas. O uso de oxigênio após extubação não se faz } \\
\text { necessário para alívio de dispneia. } \\
\text { Conclusão: A retirada da VMI reflete grande dificuldade física e emocional por parte dos profissionais, requerendo planejamento } \\
\text { cuidadoso, principalmente quando é realizado na UTI. }\end{array}$ \\
\hline Delaney e Downar, 2016 & $\begin{array}{l}\text { Identificar estudos } \\
\text { publicados sobre decisões } \\
\text { de suspender terapias de } \\
\text { manutenção de vida }\end{array}$ & Revisão Narrativa & $\begin{array}{l}\text { A preparação para a suspensão ou retirada da SAV deve iniciar com a transferência do paciente para um local privativo, sem } \\
\text { ruídos. Permitir a cultivação religiosa; orientar e discutir com a família as incertezas após retirada do SAV em relação ao tempo de } \\
\text { sobrevivência do paciente. } \\
\text { A equipe deve ser composta por médico, enfermeiro, fisioterapeuta respiratório. O processo deve se iniciar com a retirada de acessos } \\
\text { e nutrição artificial, sendo por último o desmame e extubação. O desmame deve iniciar com redução gradual da oferta de oxigênio. } \\
\text { Para a retirada da VMI, deve-se deixar em prontidão medicações opióide para alívio de sintomas e agitação, usando escalas } \\
\text { validadas para delirium e agitação no manejo. A família deve ser amparada. } \\
\text { Ainda não há evidências se os SAV devem ser retirados simultaneamente ou seguindo uma sequência específica, bem como não há } \\
\text { associação da extubação com o tempo de sobrevivência. } \\
\text { Conclusão: Existe variações na literatura sobre as práticas de retiradas da SAV, devido à falta de estudos e evidências de alta } \\
\text { qualidade. }\end{array}$ \\
\hline Oczkowski et al., 2016 & $\begin{array}{l}\text { Determinar o efeito } \\
\text { de ferramentas de } \\
\text { comunicação estruturada } \\
\text { para tomadas de decisões } \\
\text { e planejamento antecipado } \\
\text { nos CFV }\end{array}$ & Revisão Sistemática & $\begin{array}{l}\text { Identificado } 46 \text { estudos randomizados e } 21 \text { observacionais, que identificaram que o uso de ferramentas de comunicação aumentou } \\
\text { a frequência das discussões sobre planejamento avançado nos cuidados, com diretivas antecipadas, aumento na concordância entre } \\
\text { as diretivas antecipadas e as ordens médicas subsequentes para o uso ou não de SAV. } \\
\text { Conclusão: O uso de ferramentas de comunicação estruturada pode aumentar os acertos nas tomadas de decisões e planejamentos } \\
\text { de CFV. }\end{array}$ \\
\hline
\end{tabular}

$\mathrm{CFV}=$ Cuidados de fim de vida; $\mathrm{CP}=$ Cuidados paliativos; $\mathrm{SAV}=$ Suporte avançado de vida; $\mathrm{VMI}=$ Ventilação mecânica invasiva; UTI= Unidade de terapia intensiva. 
gradual, deve ser realizado cuidadosamente. ${ }^{10}$

Os resultados do presente estudo trazem o uso de opióides como medida farmacológica para alivio e manejo de agitação e dispneia, e, posicionamento e fisioterapia respiratória, como medidas não farmacológicas. ${ }^{11,14}$ Esses achados corroboram com ANCP, ${ }^{3}$ que descreve o uso de opioides e enfatiza a importância da adesão de medidas não farmacológicas, como fisioterapia, no manejo da dor e dispneia, e ressalta a importância da especialidade na equipe interdisciplinar em CP frente aos objetivos de promover controle de desconforto, dispneia e hipersecreção de vias aéreas. ${ }^{3}$

Em relação ao uso de oxigenioterapia, houve divergências entre os autores analisados e citados no presente estudo: Coelho e Yankaskas (2017), ${ }^{10}$ estabelecem em seu estudo, o uso de oxigenioterapia após extubação paliativa para o controle de dispneia. Em contrapartida, Bodnar et al. ${ }^{13}$ refutam tal achado, descrevendo que o uso de oxigenioterapia após a extubação paliativa não possui melhora significativa da dispneia, ${ }^{13}$ corroborando com a ANCP, que salienta falta de evidências quanto ao o uso de oxigênio para diminuir a dispneia. ${ }^{3}$

De forma ampla, os autores referenciados no presente estudo, defendem que VMI, nutrição artificial e diálise não melhoram o desfecho de pacientes em fase terminal, propiciam desconforto e são marcadores de CFV de baixa qualidade, ${ }^{10,18,21,23,25}$ corroborando com ANCP. ${ }^{3}$

O tema CP em UTI ainda é considerado "tabu", gera insegurança e enfrenta alguns paradigmas quanto ao seu princípio de cuidado em disparidade com os cuidados realizados em UTI, que visa o método curativo. É importante salientar que CP são uma especialidade nova e vem ganhando espaço devido ao perfil populacional do país e ao aumento das doenças crônicas, sendo necessário discutir suas abordagens juntamente com os avanços tecnológicos na área da saúde. O presente estudo apresenta limitações em relação à discussão dos resultados devido à carência de estudos relacionados aos CP e seu manejo em UTI.

\section{CONCLUSÃO}

A abordagem dos CP tem impacto positivo na UTI, com diminuição no uso de SAV e aumento na qualidade dos CFV prestados aos pacientes que não possuem possibilidade de cura, porém enfrenta limitações relacionados à falta de estudos de maior impacto, assim como protocolos assistenciais pautados em evidências, gerando dificuldade dos profissionais de saúde em estabelecer condutas paliativistas.

A UTI ainda adota seu papel curativo por meio de medidas invasivas de alta tecnologia, sendo necessária a adoção da especialidade de $\mathrm{CP}$, auxiliando a equipe multiprofissional da UTI nas tomadas de decisões e planejamento dos cuidados de pacientes com prognóstico incurável.

\section{REFERÊNCIAS}

1. Associação de Medicina Intensiva Brasileira (AMIB). Regulamento técnico para funcionamento de unidades de terapia intensiva [documento na internet]. São Paulo: AMIB; 2009 [atualizado em 30 de agosto de 2018; citado em 30 de agosto de 2018]. Disponível em: http://www.amib.org.br/noticia/nid/ recomendacoes-da-amib/

2. Machado KDG, Pessini L, Hossne WS. A formação em cuidados paliativos da equipe que atua em unidade de terapia intensiva: um olhar da bioética. Revista 2007;1(1): 34-42.

3. Academia Nacional de Cuidados Paliativos (ANCP). Manual de cuidados paliativos ANCP: $2^{\mathrm{a}}$ edição [documento na internet]. São Paulo: ANCP; 2012 [atualizado em 15 de setembro de 2018; citado em 15 de setembro de 2018]. Disponível em: http:// biblioteca.cofen.gov.br/wp-content/uploads/2017/05/Manualde-cuidados-paliativos-ANCP.pdf

4. Felix ZC, Costa SFG, Alves AMPM, Duarte MCS, Brito FM. Eutanásia, distanásia e ortotanásia: revisão integrativa da literatura. Ciência e Saúde Coletiva 2013 18(9): 2733-2746. doi: https://dx.doi.org/10.1590/S1413-81232013000900029

5. Silva EP, Sudigursky D. Concepções sobre cuidados paliativos: revisão bibliográfica. Acta Paulista de Enfermagem 2008;21(3):504-8.

6. Organização Mundial da Saúde (OMS). National cancer control programmes: policies and managerial guidelines, $2^{\mathrm{a}}$ edição [documento na internet]. Ginebra: OMS; 2002 [atualizado em 30 de outubro de 2018; citado em 30 de outubro de 2018]. Disponível em: https://www.who.int/cancer/media/en/408.pdf

7. The Economist Intelligence Unit. The 2015 Quality of Death Index Ranking palliative care across the world [documento na internet]. Lonfres: Lien foundation; 2015 [atualizado em 04 de novembro de 2018; citado em 04 de novembro de 2018]. Disponível em: https://eiuperspectives.economist.com/sites/ default/files/2015\%20EIU\%20Quality\%20of\%20Death $\% 20$ Index $\% 20$ Oct $\% 2029 \% 20$ FINAL.pdf

8. Gulini JHMB, Nascimento ERP, Moritz RD, Rosa LM, Silveira NR, Vargas MAO. A equipe da Unidade de Terapia Intensiva frente ao cuidado paliativo: discurso do sujeito coletivo. Revista da Escola de Enfermagem da USP 2017;51(e03221):1-6. doi: http://dx.doi.org/10.1590/s1980-220x2016041703221

9. Cook D, Rocker G. Dying with Dignity in the Intensive Care Unit. The New England Journal of Medicine 2014;370(26):250614. doi: http://dx.doi.org/10.1056/NEJMra1208795

10. Coelho CBT, Yankaskas JR. Novos conceitos em cuidados paliativos na unidade de terapia intensiva. Revista Brasileira de Terapia Intensiva 2017;29(2):222-30. doi: https://dx.doi. 
org/10.5935/0103-507x.20170031

11. Oczkowski SJW, Chung H, Hanvey L, Mbuagbaw L, You JJ. Communication tools for end-of-life decision-making in the intensive care unit: a systematic review and meta-analysis. Critical Care 2016;20(97):1-19. doi: http://dx.doi.org/10.1186/ s13054-016-1264-y

12. Santos DCL, Silva MM, Moreira MC, Zepeda KGM, Gaspar RB. Planejamento da assistência ao paciente em cuidados paliativos na terapia intensiva oncológica. Acta Paulista de Enfermagem 2017;30(3):295-300. doi: https://dx.doi. org/10.1590/1982-0194201700045

13. Bodnar J. Terminal Withdrawal of Mechanical Ventilation: A Hospice Perspective. Journal of Intensive Care Medicine 2018;20(10):01-09.

14. Delaney JW, Downar J. How is life support withdrawn in intensive care units: A narrative review. Journal of Critical Care 2016;35:12-8. doi: https://dx.doi.org/10.1016/j.jcrc.2016.04.006 15. Silva RS, Campos ERA, Pereira A. Cuidando do paciente no processo de morte na unidade de terapia intensiva. Revista da Escola de Enfermagem da USP 2011; 45(3):738-44. doi: http:// dx.doi.org/10.1590/S0080-62342011000300027

16. Gulini JHMB, Nascimento ERP, Moritz RD, Vargas MAO, Matte DL, Cabral RP. Fatores preditores de óbito em Unidade de Terapia Intensiva: contribuição para a abordagem paliativista. Revista da Escola de Enfermagem da USP 2018;52(e3342):1-7. doi: http://dx.doi.org/10.1590/s1980-220x2017023203342

17. Binney ZO, Quest TE, Feingold PL, Buchman T, Majesko AA. Feasibility and Economic Impact of Dedicated Hospice Inpatient Units for Terminally Ill ICU Patients. Critical Care Medicine 2014;42(5):1074-80. doi: http://dx.doi.org/10.1097/ CCM.0000000000000120

18. Nakagawa S, Garan AR, Takayama H, Takeda K, Topkara VK, Yuzefpolskaya M, Lin SX, Colombo PC, Naka Y, Blinderman CD. End of Life with Left Ventricular Assist Device in Both Bridge to Transplant and Destination Therapy. Journal of Palliative Medicine 2018;21(9):1284-1289. doi: http://dx.doi. org/10.1089/jpm.2018.0112

19. Wright AA, Zhang B, Keating NL, Weeks JC, Prigerson HG. Associations between palliative chemotherapy and adult câncer patients' end of life care and place of death: prospective cohort study. British Medical Journal 2014;348:g1219. doi: http:// dx.doi.org/10.1136/bmj.g1219

20. Gu X, Chen M, Liu M, Zhang Z, Cheng W. End-of-life decision-making of terminally ill cancer patients in a tertiary cancer center in Shanghai, China. Support Care Cancer 2016;24(5):2209-15. doi: http://dx.doi.org/10.1007/s00520015-3017-X

21. Koff G, Vaid U, Len E, Crawford A, Oxman DA. Differences in Utilization of Life Support and End-of-Life Care for Medical ICU Patients With Versus Without Cancer. Critical Care Medicine 2017;45(4):e379-83. doi: http://dx.doi.org/10.1097/ CCM.0000000000002260

22. Mazutti SRG, Nascimento AF, Fumis RRL. Limitação de Suporte Avançado de Vida em pacientes admitidos em unidade de terapia intensiva com cuidados paliativos integrados. Revista
Brasileira de Terapia Intensiva 2016;28(3):294-300. doi: https:// dx.doi.org/10.5935/0103-507X.20160042

23. Huang HK, Hsieh JG, Hsieh CJ, Wang YW. Do cancer patients with dementia receive less aggressive treatment in end-of-life care? A nationwide population-based cohort study. Oncotarget 2017;8(38): 63596-604. doi: https://dx.doi. org/10.18632/oncotarget. 18867

24. Santos EAA. Barreiras associadas aos cuidados na demência: uma revisão da literatura. Geriatrics, Gerontology and Aging 2018;12(2):105-12.

25. Hua M, Ma X, Morrison SR, Li G, Wunsch H. Association between the Availability of Hospital-Based Palliative Care and Treatment Intensity for Critically Ill Patients. Annals of the American Thoracic Society 2018;15(9):1067-74. doi: https:// dx.doi.org/10.1513/AnnalsATS.201711-872OC
Recebido em: 28/06/2020

Aceito em:07/12/2020

Como citar: BARRETO, Tiago Lima et al. Cuidados paliativos em terapia intensiva: uma revisão integrativa de literatura. Revista Interdisciplinar de Promoção da Saúde, Santa Cruz do Sul, v. 3, n. 2, maio 2021. ISSN 2595-3664. Disponível em: <https://online. unisc.br/seer/index.php/ripsunisc/article/view/15378 >. Acesso em: 30 maio 2021. doi:https://doi.org/10.17058/rips.v3i2.15378 